'Department of Ophthalmology, Retina Eye Hospital, Bursa, Turkey

${ }^{2}$ Department of Ophthalmology, Maltepe University Medical School, Istanbul, Turkey

${ }^{3}$ Department of Ophthalmology, Gazi University Medical School, Ankara, Turkey

${ }^{4}$ Department of Ophthalmology, Ulucanlar Eye Education and Research Hospital, Ankara, Turkey

${ }^{5}$ Department of Ophthalmology, Adana Retina Eye Center, Adana, Turkey

${ }^{6}$ Department of Ophthalmology, Sisli Etfal Education and Research Hospital, Istanbul, Turkey

${ }^{7}$ Department of Ophthalmology, Uludag University Medical School, Bursa, Turkey

${ }^{8}$ Department of Ophthalmology, Gulhane Military Medical Academy, Ankara, Turkey

${ }^{9}$ Private Practice, Ankara, Turkey

${ }^{10}$ Department of Ophthalmology, International Kolan Hospital, Istanbul, Turkey

'Department of Ophthalmology, Saint Louis University Medical School,

Missouri, USA

${ }^{12}$ Department of Ophthalmology, Dokuz Eylul University Medical School, Izmir, Turkey

${ }^{13}$ Department of Biostatistics, Kocatepe iversity Medical Schoo Afyonkarahisar, Turkey

${ }^{14}$ Department of Ophthalmology, Kocatepe University Medical School Afyonkarahisar, Turkey

Correspondence:

R Avci, Ophthalmology, Retina Eye Hospital, Sanayi Cad No:171, Nilufer، Bursa 16600, Turkey

Tel: +905323971719 or

+902242402400 0102

Fax: +902242402403.

E-mail: ravci@bursaretina.com

This study was presented in part as a free paper in EURETINA meeting in Hamburg, 2013, at Turkish-Israel Joint Meeting in EURETINA meeting in Hamburg, 2013 and presented in part as a scientific poster at the 31 st Annual Meeting of American Society of Retinal Specialists, 24-28 August 2013, Toronto, ON, Canada.

Received: 7 November 2016 Accepted in revised form: 19 June 2017 Published online: 21 July 2017

\title{
Multicenter study of pars plana vitrectomy for optic disc pit maculopathy: MACPIT study
}

\begin{abstract}
Purpose To evaluate surgical intervention with pars plana vitrectomy (PPV) for correction of optic disc pit maculopathy (ODP-M).

Patients and methods Retrospective chart review from 13 centres of 51 eyes of 50 patients with ODP-M who underwent PPV between 2002-2014. Anatomic and final bestcorrected visual acuity (BCVA) outcomes were evaluated for all cases with different adjuvant techniques.

Results There were 23 males and 27 females with median age 25.5 (6-68) years.

Preoperative median foveal thickness was 694.5 (331-1384) $\mu \mathrm{m}$ and improved to 252.5 (153-1405) $\mu \mathrm{m}$. Median BCVA improved from $20 / 200(20 / 20000$ to $20 / 40)$ to $20 / 40(20 / 2000$ to 20/20) with 20/40 or better in 31 eyes. Complete retinal reattachment was achieved in 44 eyes $(86.3 \%)$ at 7.1 (5.9) months. The good surgical outcomes were achieved in different adjuvant groups. Median follow-up was 24 (6 to 120) months.

Conclusions These results confirm the longterm effectiveness of PPV for ODP-M.

Prospective studies are needed to determine the effectiveness of any adjuvant technique in improving the success of PPV for ODP-M. Eye (2017) 31, 1266-1273; doi:10.1038/eye.2017.142; published online 21 July 2017
\end{abstract}

\section{Introduction}

Optic disc pits (ODPs) have long been recognized as congenital abnormalities of the optic nerve head. ${ }^{1}$ The prevalence of congenital ODP is estimated at 1 in 11000 ophthalmic patients. ${ }^{1,2}$ Males and females are affected equally. Optic disc pits are usually unilateral, but may occasionally occur in both eyes. ${ }^{1}$ In isolated cases without macular involvement, prognosis is good with normal visual acuity
R Avci ${ }^{1}$, Z Kapran², Ş Ozdek³ ${ }^{3}$ MY Teke ${ }^{4}$, O Oz ${ }^{5}$,

D Guven ${ }^{6}$, S Yilmaz' ${ }^{1}$, B Kaderli ${ }^{7}$, AH Durukan ${ }^{8}$,

G Sobaci ${ }^{9}$, YB Unver ${ }^{10}$, L Akduman ${ }^{11}$,

S Kaynak ${ }^{12}$, I Dogan $^{13}$ and UU Inan ${ }^{14}$ except an arcuate scotoma or enlarged blind spot in some cases. However, vision deteriorates if optic disc pit maculopathy (ODP-M) consisted mainly of serous macular detachment or macular schisis develops. ${ }^{2-6}$

Although pars plana vitrectomy (PPV) is generally accepted as the standard procedure for the management of ODP-M, the need for various adjuvant therapies has not been well studied. ${ }^{4-10}$ The current study was designed in an attempt to pool the data from multiple centers due to the rarity of this condition and the need for larger numbers to determine effective treatment methods.

\section{Materials and methods}

This multicentre investigation (MACPIT Study) consists of interventional consecutive cases from 13 tertiary centres. The study was organized by the Society of Vitreoretinal Surgery of the Turkish Ophthalmological Association. A total of 51 eyes were included in this retrospective interventional case study. The patients were treated with a PPV procedure between January 2002 and December 2014. The patients selected for inclusion in the study had complete optical coherence tomography (OCT) recordings and follow-up of at least 6 months. The main outcome measures were complete reattachment of the macula, improvement in central macular thickness (CMT) and visual acuity.

\section{Procedure}

After core vitrectomy, the posterior hyaloid was removed. Triamcinolone acetonide (TA) was used to ensure the complete removal of any vitreous residue in 28 eyes. Fluid-air exchange was performed, followed by gas tamponade with sulphur hexafluoride (SF6) or perfluoropropane gas (C3F8). Postoperative 
facedown positioning for $\sim 1$ week was recommended by all surgeons. Thirty-four patients had C3F8 and 13 had SF6 and 2 had C2F6 gas tamponade. ILM peeling was performed in 18 eyes and JLP was performed in 35 eyes.

\section{Categorical grouping of collected cases}

A record was made of the use of intraoperative triamcinolone for better visualization and removal of the vitreous, the application of $180^{\circ}$ juxtapapillary endolaser photocoagulation (JLP) in mild-to-moderate intensity to the retina in the temporal margin of the optic disc prior to the fluid-air exchange, implementation of internal limiting membrane (ILM) peeling, and the type of intraocular tamponade as well as intraoperative events and complications. The patients were then divided into four main categories according to the adjunctive techniques. Anatomical and functional results were evaluated in the following groups: C3F8 or SF6 tamponade group, JLP or no JLP application group, ILM peeling or no ILM peeling group, and intraoperative TA use or no TA use group.

\section{Data review}

The clinical features, surgical characteristics, surgical and visual outcomes of the collected cases were analysed. The preoperative data were collected for demographics (age and gender), laterality, presenting visual symptoms and best-corrected visual acuity. The postoperative data included the type of surgery performed, intraocular or postoperative complications, repeated surgery and followup examinations. All the data were collected via a retrospective chart review. Optical coherence tomography was performed before and at each visit after the surgery. Spectral domain OCT was used in the majority of cases. If the data were obtained by Time Domain OCT, $50 \mu \mathrm{m}$ was added to the CMT. ${ }^{11}$ A reduction of more than half of baseline CMT over time was used as a marker of partial resolution or partial anatomical success. The disappearance of all the fluid in retinal compartments was defined as complete resolution (CR) or complete anatomical success. Best-corrected Snellen visual acuities were recorded. Snellen visual acuities were converted to the logarithm of the minimum angle of resolution (logMAR) scale for statistical analysis. Informed consent for surgery was obtained from each patient after providing a complete explanation of PPV procedure and natural course of the ODP-M. The study was conducted in accordance with the ethical principles represented in the Declaration of Helsinki. Kocatepe University Clinical Research Ethics Committee reviewed and approved the study.

Statistical analysis was applied with SPSS 20.0 for Windows package. Normality of the data was tested with Kolmogorov-Smirnov test with Lilliefors significance correction. The Friedmann test was used to detect statistical differences between the repeated measurements. All tests were two-sided. Descriptive data were summarised as median and range if the data are not normally distributed. A value of $P<0.05$ was considered statistically significant.

\section{Results}

The data charts of 51 eyes of 50 patients with ODP-M undergoing PPV were reviewed. The patients were 23 males and 27 females with median age of 25.5 years ranging in age from 6 to 68 years. All patients had noted a central scotoma or metamorphopsia, and a decline in Snellen visual acuity was observed in all eyes prior to surgical intervention. The right eye was affected in 28 patients, the left eye in 21 patients and 1 patient had bilateral ODP-M. Characteristics of the all eyes were summarized in Table 1.

An OCT evaluation of the eyes before surgery revealed double-layer separation in 42 eyes, pure intraretinal separation in two eyes, serous macular detachment (SMD) in 10 eyes and outer layer hole in 8 eyes. One eye had a lamellar macular hole at baseline. The time from the onset of visual symptoms to surgery ranged from 2 weeks to 60 months (mean \pm SD: 11.7 (16.3) months; median: 2.75 months). The vitreoretinal technique was 23-gauge in 34 eyes, 20-gauge in 11 eyes and 25-gauge in 6 eyes.

Complete resolution (CR) was observed in 44 of 51 eyes (86.3\%). The time to CR was mean (SD) $7.1 \pm 5.9$ months and median 5 months (range, 1-20 months). Thirty eyes $(58.8 \%)$ showed CR within 6 months, while 13 eyes $(25.5 \%)$ resolved at month-12 and thereafter. Complete resolution was determined at 18 months or later in 4 eyes (Figure 1) and 5 eyes showed partial resolution. Central macular thickness did not decrease in 2 eyes. Of the five eyes that showed failure to CR, three had 12-month follow-up. Preoperative median BCVA and CMT improved from $20 / 200$ and $700 \mu \mathrm{m}$ to $20 / 40$ and $257 \mu \mathrm{m}$ at the final visit, respectively. A gain of 2 or more Snellen lines of vision was determined in 43 (84\%) eyes and 31 (61\%) reached to 20/40 or better visual acuity. A mean (SD) improvement of 6.19 (5.82) lines by ETDRS chart was obtained. Improvement in BCVA and CMT was statistically significant $(P<0.001)$. Initial visual acuity stabilized or improved in all except two eyes. Both of these eyes had undergone a second intervention, for macular hole in one eye and for recurrence in the other. Mean follow-up was 32.8 months (median: 24 months, range: $12-120$ months).

When evaluating each adjuvant group for functional and anatomical success, all categories resulted in statistically significant improvements at month-12 and the final visit. The complete resolution rate, mean time to $C R$, and improvement in CMT or BCVA at month-12 or final 
Table 1 Summarised description of all eyes undergoing pars plana vitrectomy with different adjuvant techniques for Optic Disc Pit Maculopathy

\begin{tabular}{|c|c|c|c|}
\hline Patient and Eye Characteristics & $\mathrm{n}$ & $\%$ & Median (Min-Max) \\
\hline Age (Years) & & & $25.5(6-68)$ \\
\hline \multicolumn{4}{|l|}{ Gender } \\
\hline Female & 27 & 54 & \\
\hline Male & 23 & 46 & \\
\hline \multicolumn{4}{|l|}{ Laterality } \\
\hline Right & 29 & 57 & \\
\hline Left & 22 & 43 & \\
\hline Preoperative Symptom Duration (Month) & & $2.75(0.5-60)$ & \\
\hline Preoperative BCVA & & & $20 / 200(20 / 40$ to $20 / 20000)$ \\
\hline Final BCVA & & & $20 / 40(20 / 20$ to $20 / 2000)$ \\
\hline Preoperative CMT $(\mu \mathrm{m})$ & & & $694.5(331-1384)$ \\
\hline Final CMT $(\mu \mathrm{m})$ & & & $252.5(153-1405)$ \\
\hline Complete resolution & 44 & 86.3 & \\
\hline Complete resolution time (month) & & & $5(1-20)$ \\
\hline \multicolumn{4}{|l|}{ Tamponades } \\
\hline $\mathrm{C}_{3} \mathrm{~F}_{8}$ & 34 & 66.7 & \\
\hline $\mathrm{SF}_{6}$ & 13 & 25.50 & \\
\hline $\mathrm{C}_{2} \mathrm{~F}_{6}$ & 2 & 3.9 & \\
\hline Silicone & 2 & 3.9 & \\
\hline \multicolumn{4}{|l|}{ Triamcinolone-assisted vitreous removal } \\
\hline Yes & 28 & 54.9 & \\
\hline No & 23 & 45.1 & \\
\hline \multicolumn{4}{|l|}{ Internal limiting membrane peeling } \\
\hline Yes & 18 & 35.3 & \\
\hline No & 33 & 64.7 & \\
\hline \multicolumn{4}{|l|}{ Juxtapapillary laser photocoagulation } \\
\hline Yes & 35 & 68.6 & \\
\hline No & 16 & 31.4 & \\
\hline \multicolumn{4}{|l|}{ Type of PPV } \\
\hline $20 \mathrm{G}$ & 11 & 21.6 & \\
\hline $23 \mathrm{G}$ & 34 & 66.7 & \\
\hline $25 \mathrm{G}$ & 6 & 11.7 & \\
\hline Follow-up (month) & & & $24(6-120)$ \\
\hline \multicolumn{4}{|l|}{ Centre } \\
\hline Bursa Retina Eye Hospital & 17 & 33.33 & \\
\hline Gazi University Hospital & 8 & 15.69 & \\
\hline Sisli Etfal Hospital & 2 & 3.92 & \\
\hline Gulhane Military Hospital & 1 & 1.96 & \\
\hline Saint Lois University Hospital & 1 & 1.96 & \\
\hline Maltepe University Hospital & 8 & 15.69 & \\
\hline Uludag University Hospital & 1 & 1.96 & \\
\hline Ulucanlar Eye Hospital & 6 & 11.77 & \\
\hline Dokuz Eylul University Hospital & 1 & 1.96 & \\
\hline Adana Retina Eye Centre & 3 & 5.88 & \\
\hline Kocatepe University Hospital & 1 & 1.96 & \\
\hline International Kolan Hospital & 1 & 1.96 & \\
\hline Private Practice & 1 & 1.96 & \\
\hline
\end{tabular}




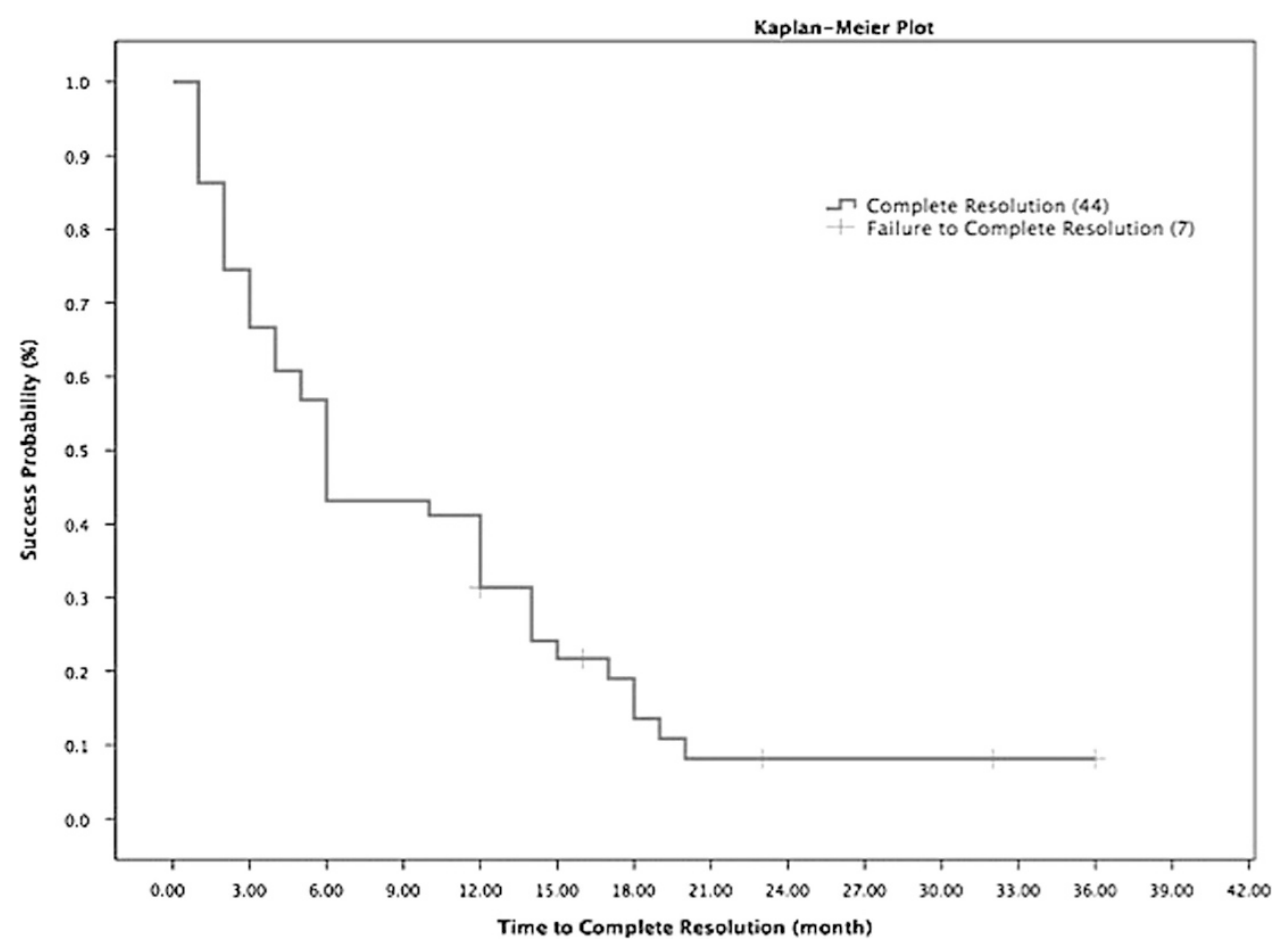

Figure 1 Kaplan-Meier Graph for the time to Complete Resolution of Optic Disc Pit Maculopathy: vertical axis shows proportion of eyes with persistent detachment and the horizontal axis shows the time (in months) to complete resolution from the date of surgery. Complete anatomical success within the first 6 months was seen in $\sim 60 \%$ of the study eyes and $25 \%$ reattached after month- 12 .

visit for all eyes and the groups (C3F8 or SF6, ILM peeling or no ILM peeling, JLP or no JLP, intraoperative TA or no TA utilization) were given in Tables 1 and 2.

Peripheral retinal tear and full-thickness macular hole developed in 2 and 4 eyes, respectively during the surgery. Three eyes underwent second surgery for macular hole repair, inferior retinal detachment and silicon-out procedure. Additional vitrectomy with gas tamponade was performed in one eye and incomplete resolution was observed in the twelveth month.

Additional JLP was performed in one eye. Lens opacities developed in two eyes, and both underwent cataract surgery during the follow-up. A representative case demonstrating slowly improvement in the long-term is shown in Figure 2.

\section{Discussion}

There are currently no guidelines for the treatment of ODP-M, and no consensus on the optimal surgical technique. ${ }^{6-14}$ Although the best functional outcomes seem to be obtained with vitrectomy, controversy continues on the rationale for the adjunctive steps taken with vitrectomy. In our study, which reports largest series to date, we observed that PPV and gas tamponade with or without JLP and ILM peeling is effective for ODP-M in majority of the cases.
Juxtapapillary Laser Photocoagulation is a somewhat controversial procedure during vitrectomy. The barrier effect of JLP for fluid transportation may have beneficial additive effects to the vitrectomy to obtain better results. ${ }^{9-11}$ It has been reported that the combination of vitrectomy plus gas tamponade plus JLP was more effective than vitrectomy and gas. ${ }^{9}$ Some authors have proposed that the rationale for JLP to prevent fluid transportation within the inner retinal layers of the macula would be weak and have suggested that laser treatment in the papillomacular region may have adverse effects. ${ }^{12}$ Recent studies have generally reported no additional benefit from the laser in long-term success rates. ${ }^{13-16}$ Our study also suggest that JLP may not be necessary for success of PPV for at least our follow-up period.

The addition of ILM peeling procedure to the vitreoretinal techniques ensures complete hyaloid removal and also targets relief of complete surface traction on the retina between the optic disc and fovea. ${ }^{12,16}$ The authors in favour of ILM peeling believe that both tangential and anteroposterior traction facilitate the passage of fluid from the optic pit into the retina and tangential traction cannot be eliminated unless the ILM is peeled. ${ }^{16-18}$ In the current series, ILM peeling was not performed except 18 eyes, and good surgical outcomes were obtained in these eyes. Some previous reports 


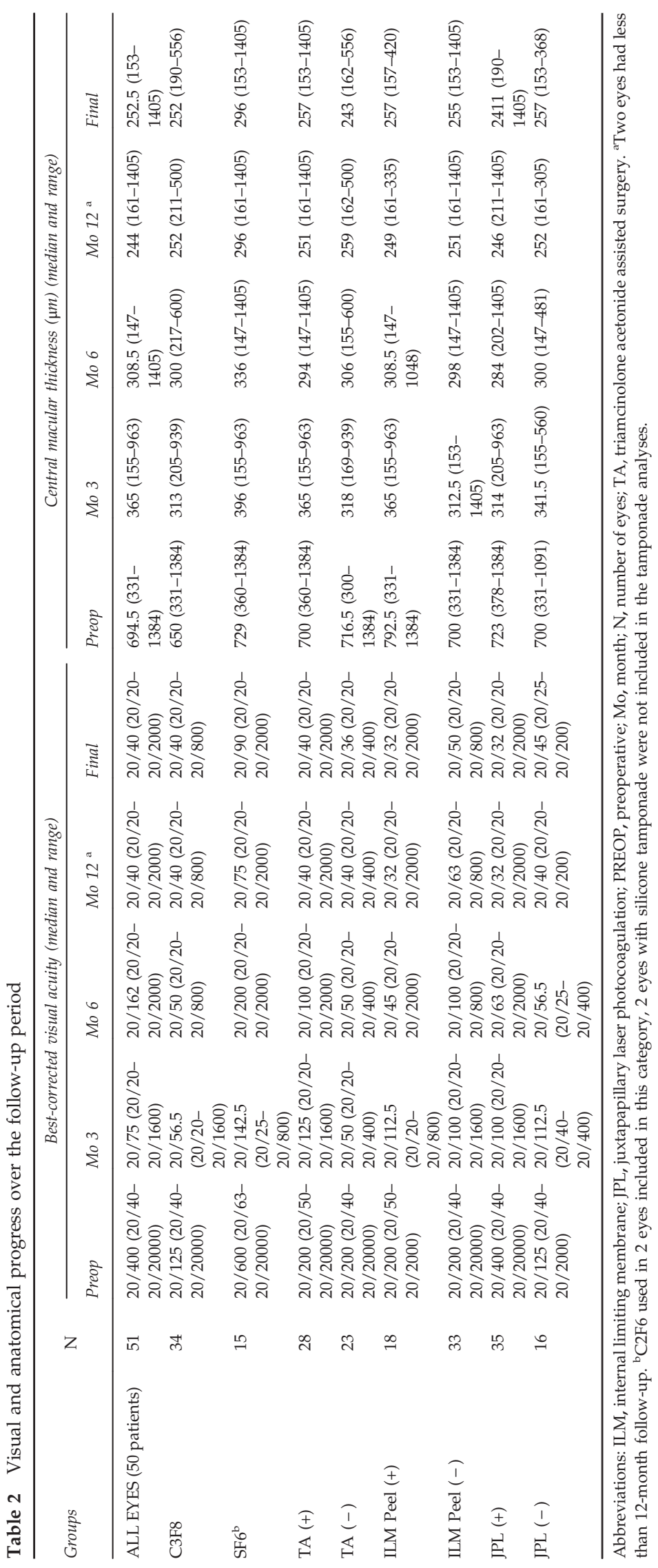



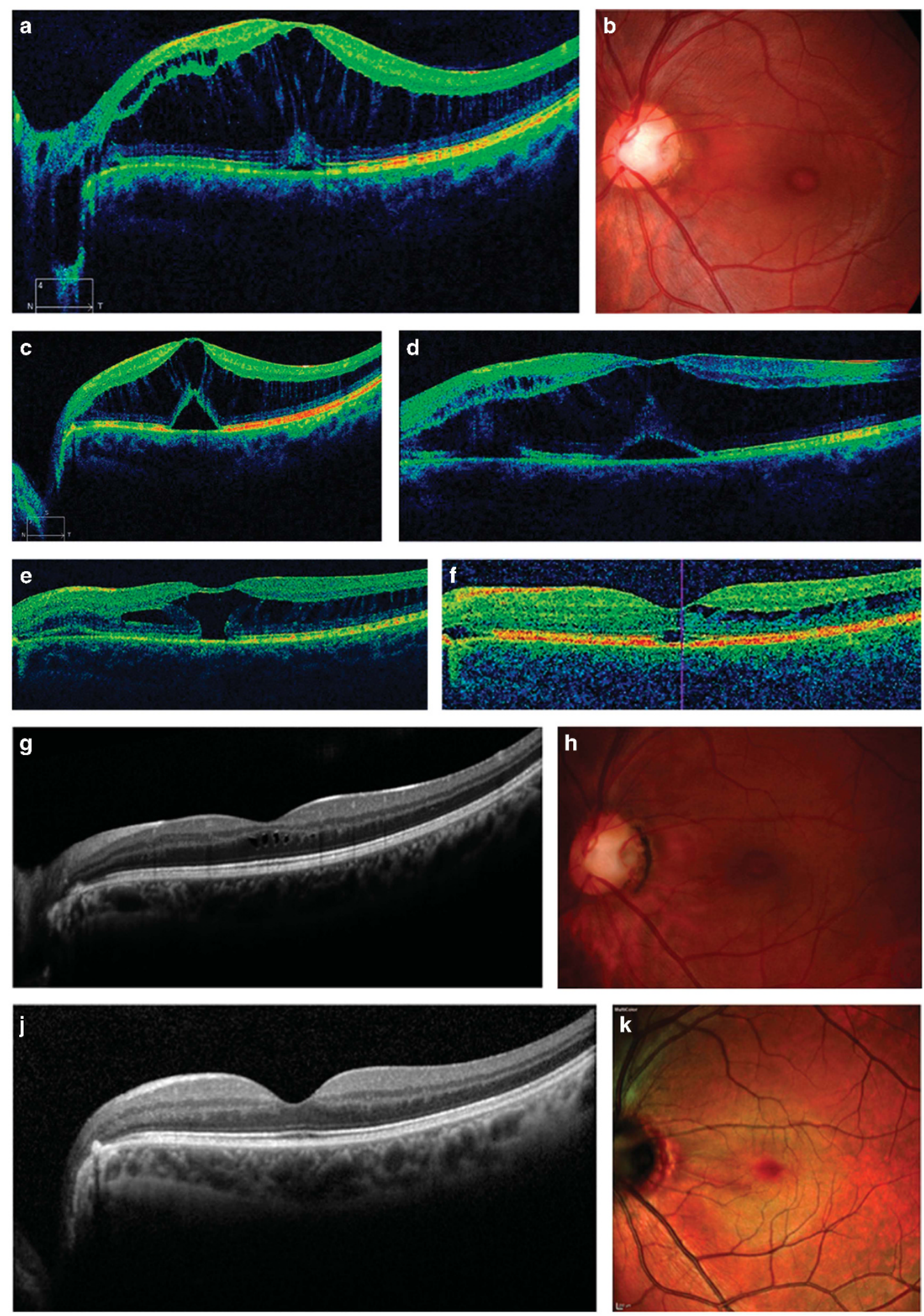

Figure 2 For caption see next page. 
Figure 2 A 19-year-old male student in the Dentistry Faculty presented with progressive visual deterioration, which had been ongoing for 3 months. ( $a$ and b) Fundus examination and OCT revealed ODP maculopathy. Visual acuity was 20/63. Due to university studies, the operation was delayed for 2 months until June 2012. PPV was applied together with juxtapapillary laser photocoagulation (JLP) and $13 \%$ C3F8 tamponade. (c) 3 months after the operation, there was little change to the macular thickness and visual acuity was the same. (d) Macular thickness decreased significantly and an outer layer hole developed at 7 months. BCVA was 20/50. (e) Macula was attached at month-12 with collapse of schisis-like intraretinal separation. There was residual intraretinal fluid with interruption of the central ellipsoid zone. BCVA was 20/40. (f and g) Intraretinal fluid gradually disappeared at months 18 and 19. BCVA was 20/32 and 20/25, respectively, at these visits. (h) Fundus photography shows an area of tiny residual subretinal fluid near the optic nerve head. ( $j$ and $k$ ) Retinal restoration was complete at month 33. BCVA was 20/20. Multicolour photography shows complete attachment with a thin and regular JPL scar. Automated visual field testing showed no related visual field scotoma. Follow-up was 42 months.

consistent with our study suggested that ILM peeling is probably not essential in the treatment of most cases of ODP-M. ${ }^{9-14}$

Intraoperative gas is also believed to displace the retinal and subretinal fluid away from the macula. ${ }^{19,20}$ A recent study has suggested that resolution of ODP maculopathy may be faster in patients with gas tamponade. ${ }^{21}$ Some recently published studies have also suggested that gas injection has no significant effect on the final success rate and if used, no type of gas has been shown to be superior to any other type. ${ }^{9,12,21}$ In the current series, C3F8 gas tamponade had been preferred in more cases than SF6 gas. Both $\mathrm{C} 3 \mathrm{~F} 8$ and $\mathrm{SF} 6$ gas tamponade were regarded to have efficient to achieve surgical success. Our results related to gas tamponade is consistent with the recently published reports. ${ }^{11-14,21}$

Triamcinolone-assisted vitrectomy, as a surgical technique has been emphasized previously for the surgical treatment of ODP-M. ${ }^{12,15}$ As posterior vitreous traction on the margin of the optic disc has been suggested to be responsible for the development of maculopathy in eyes with ODP, complete removal of the posterior hyaloid without residues attached to ILM may be important for surgical success. ${ }^{22,23}$ Thus, intraoperative TA can ensure to achieve this goal. However, in the current series, anatomic and visual outcomes were favourable in the cases with triamcinolone-assisted vitrectomy and other cases.

The complete macular reattachment rate within the follow-up period of the current study was $86.3 \%$, which is similar to previously reported case series. ${ }^{6-18}$ A delay in complete reabsorption of macular fluid beyond 12 months has been described in literature. ${ }^{6,10-14}$ In the current series, 13 eyes showed complete resolution at 12 months or longer, and 4 eyes at month 18 and thereafter. Therefore, in cases with incomplete resolution, a follow-up period of at least 12 months may be necessary before the second intervention is considered. In the current series, three out of seven eyes with partial resolution had 12 months follow-up and it is possible that resolution would improve further with additional time in these eyes.

However, there have been reports of recurrent cases even 16 years after the last vitrectomy. ${ }^{24}$ Different factors such as vitreous traction and difficulty in reabsorption of viscous subretinal fluid in untreated eyes have been suggested to cause the persistence of ODP-M, but no verified factor is known as a reason for persistent fluid after vitreoretinal surgery. In recurrent cases, the underlying pathology may be related to cerebrospinal fluid forced by gradient pressure.

Our series were collected from the long-term time interval in which a variety of surgical techniques and steps increased. Comparing these different adjuvant techniques is quite difficult because making groups in sufficient size is almost impossible in a prospective fashion due the rare nature of optic pit maculopathy. Therefore, due to retrospective nature of the study and insufficient size of the groups, we did not perform statistical analysis of the groups to find out superiority of any adjuvant technique.

In conclusion, PPV with gas tamponade with or without JLP can be considered to be good approaches to optimize surgical outcomes. There is still a need for prospective and comparative multicentre studies collecting case series with larger sample sizes to evaluate the efficacy of adjunct techniques combined with vitrectomy.

\section{Summary}

What was known before

- Pars plana vitrectomy has been preferred method for surgical treatment of optic disc pit maculopathy (ODP-M).

- There are several adjuvant procedures in combination with pars plana vitrectomy.

- There is no consensus about benefit of any adjuvant procedure.

What this study adds

- This study conducts largest case series ever published in the literature.

- This study confirms the effectiveness of pars plana vitrectomy for optic disc pit maculopathy.

- PPV and any type of gas tamponade with or without other adjuvant techniques are successful for surgical management of ODP-M in majority of cases.

- This study suggest surgical success is possible without ILM peeling or JLP. 


\section{Conflict of interest}

The authors declare no conflict of interest.

\section{References}

1 Apple DJ, Rabb MF, Walsh PM. Congenital anomalies of the optic disc. Surv Ophthalmol 1998; 27: 3-41.

2 Postel EA, Pulido JS, McNamara JA, Johnson MW. The etiology and treatment of macular detachment associated with optic nerve pits and related anomalies. Trans Am Ophthalmolol Soc 1998; 96: 73-93.

3 Gass JDM. Serous detachment of the macula secondary to congenital pit of the optic nerve head. Am J Ophthalmol 1969; 67: 821-841.

4 Jain N, Johnson MW. Pathogenesis and treatment of maculopathy associated with cavitary optic disc anomalies. Am J Ophthalmol 2014; 158: 423-435.

5 Gowdar JP, Rajesh B, Giridhar A, Gopalakrishnan M, Hussain R, Thachil T. An insight into the pathogenesis of optic disc pit-associated maculopathy with enhanced depth imaging. JAMA Ophthalmol 2015; 133: 466-469.

6 Georgalas I, Ladas I, Georgopoulos G, Petrou P. Optic disc pit: a review. Graefes Arch Clin Exp Ophthalmol 2011; 249: 1113-1122.

7 Garcia-Arumi J, Guraya BC, Espax AB, Castillo VM, Ramsay LS, Motta RM. Optical coherence tomography in optic pit maculopathy managed with vitrectomy-laser-gas. Graefe's Arch Clin Exp Ophthalmol 2004; 242: 819-826.

8 Cox MS, Witherspoon CD, Morris RE, Flynn HW. Evolving techniques in the treatment of macular detachment caused by optic nerve pits. Ophthalmology 1988; 95: 889-896.

9 Moisseiev E, Moisseiev J, Loewenstein A. Optic disc pit maculopathy: when and how to treat? A review of the pathogenesis and treatment options. Int J Retin Vitr 2015; 1: $1-9$.

10 Avci R, Yilmaz S, Inan UU, Kaderli B, Kurt M, Yalcinbayir O et al. Long-term outcomes of pars plana vitrectomy without internal limiting membrane peeling for optic disc pit maculopathy. Eye 2013; 27: 1359-1367.

11 Abouammoh MA, Alsulaiman SM, Gupta VS, Mousa A, Hirakata A, Berrocal MH et al. King Khaled Eye Specialist Hospital International Collaborative Retina Study Group Pars plana vitrectomy with juxtapapillary laser photocoagulation versus vitrectomy without juxtapapillary laser photocoagulation for the treatment of optic disc pit maculopathy: the results of the KKESH International Collaborative Retina Study Group. Br J Ophthalmol 2016; 100: 478-483.

12 Hirakata A, Inoue M, Hiraoka T, McCuen BW 2nd. Vitrectomy without laser treatment or gas tamponade for macular detachment associated with an optic disc pit. Ophthalmology 2012; 119: 810-818.

13 Steel DH, Williamson TH, Laidlaw DA, Sharma P, Matthews C, Rees J et al. Extent and location of intraretinal and subretinal fluid as prognostic factors for the outcome of patients with optic disk pit maculopathy. Retina 2016; 36: 110-118.

14 Rayat JS, Rudnisky CJ, Waite C, Huang P, Sheidow TG, Kherani A et al. Long-Term outcomes for optic disk pit maculopathy after vitrectomy. Retina 2015; 35: 2011-2017.

15 Hirakata A, Okada AA, Hida T. Long-term results of vitrectomy without laser treatment for macular detachment associated with an optic disc pit. Ophthalmology 2005; 112: 1430-1435.

16 Shukla D, Kalliath J, Tandon M, Vijayakumar B. Vitrectomy for optic disk pit with macular schisis and outer retinal dehiscence. Retina 2012; 32: 1337-1342.

17 Gandorfer A, Kampik A. Role of vitreoretinal interface in the pathogenesis and therapy of macular disease associated with optic pits. Ophthalmologe 2000; 974: 276-279.

18 Rizzo S, Belting C, Genovesi-Ebert F, Di Bartolo E, Cresti F, Cinelli L et al. Optic disc pit maculopathy: the value of smallgauge vitrectomy, peeling, laser treatment, and gas tamponade. Eur J Ophthalmol 2012; 22: 620-625.

19 Lincoff H, Yannuzzi L, Singerman L, Kreissig I, Fisher Y. Improvement in visual function after displacement of the retinal elevations emanating from optic pits. Arch Ophthalmol 1993; 111: 1071-1079.

20 Johnson TM, Johnson MW. Pathogenic implications of subretinal gas migration through pits and atypical colobomas of the optic nerve. Arch Ophthalmol 2004; 122: 1793-1800.

21 Teke MY, Citirik M. 23 Gauge vitrectomy, endolaser, and gas tamponade versus vitrectomy alone for serous macular detachment associated with optic disc pit. Am J Ophthalmol 2015; 160: 779-785.

22 Gregory-Roberts EM, Mateo C, Corcóstegui B, Schiff WM, Chang LK, Quiroz-Mercado H et al. Optic disk pit morphology and retinal detachment: optical coherence tomography with intraoperative correlation. Retina 2013; 33: 363-370.

23 Matsumoto H, Yamanaka I, Hisatomi T, Enaida H, Ueno A, Hata $\mathrm{Y}$ et al. Triamcinolone acetonide-assisted pars plana vitrectomy improves residual posterior vitreous hyaloid removal: ultrastructural analysis of the inner limiting membrane. Retina 2007; 27: 174-179.

24 Coca MN, Tofigh S, Elkeeb A, Godley BF. Optic disc pit maculopathy recurring in the absence of vitreous gel. JAMA Ophthalmol 2014; 132: 1375-1376. 